\title{
Reaction-diffusion equations in a noncylindrical thin domain
}

\author{
Jamil V Pereira and Ricardo P Silva*
}

\section{"Correspondence:}

rpsilva@rc.unesp.br

Departamento de Matemática, Instituto de Geociências e Ciências

Exatas, UNESP - Universidade

Estadual Paulista, Rio Claro,

13506-900, Brazil

\begin{abstract}
In this paper we are concerned with nonlinear reaction-diffusion equations posed in a time-dependent family of domains $\left\{\Omega_{t}^{\epsilon}\right\}_{t \in \mathbb{R}} \subset \mathbb{R}^{n+1}$ which collapses to a lower dimensional set as the parameter $\epsilon$ goes to 0 .

MSC: Primary 35K58; secondary 35B41
\end{abstract}

Keywords: evolution process; thin domains; noncylindrical domain

\section{Introduction}

Evolutionary PDEs on time-varying domains have been the subject of intense research over the past few years; we can cite, for example, [1-6] and the references given therein. Fundamental questions such as existence, regularity, and the asymptotic behavior of solutions are frequent topics of study and, since such problems are intrinsically nonautonomous, they need to be considered in a non-autonomous setting. These problems generate evolution process, and we can indicate the references $[7,8]$ which include a substantial overview on this topic.

Inspired by the recent works $[4,5]$, both of them related to reaction-diffusion equations on time-dependent domains, in this paper we are concerned with reaction-diffusion equations in a time-dependent thin domain. To the best of our knowledge, this is an untouched topic in the literature. We are going to prove that the evolution process generated by a family of singularly perturbed reaction-diffusion equations, which is equivalent in the autonomous case to the flow generated by such a family, converges to the evolution process generated by a limiting equation posed in a lower dimensional domain. This is the first step in order to consider the continuity of asymptotic dynamics (attractors), which will be considered in a further work.

One of the pioneering works on (autonomous) nonlinear reaction-diffusion equations on thin domains was [9], where the authors had considered one reaction-diffusion equation posed in the ordinate set under a smooth function $g:\left[0, \epsilon_{0}\right] \times \mathbb{R}^{n} \rightarrow \mathbb{R}^{+}$satisfying $g(\epsilon, x) \stackrel{\epsilon \rightarrow 0}{\longrightarrow} 0$ (uniformly in $x$ ). They found a limiting equation $(\epsilon=0)$ ), which was defined in a lower dimensional domain, and showed that the flow generated by such an equation behaves continuously (with respect to $\epsilon$ in $H^{1}$-norms). Our aim in this paper is to prove a similar result for the evolution process generated by equations on time-dependent domains.

In order to set up the problem, let $\omega$ be a smooth bounded domain in $\mathbb{R}^{n}, n \geq 1$, and $g \in C^{2}(\bar{\omega} \times \mathbb{R} ; \mathbb{R})$ satisfying

( 2013 Pereira and Silva; licensee Springer. This is an Open Access article distributed under the terms of the Creative Commons Attribution License (http://creativecommons.org/licenses/by/2.0), which permits unrestricted use, distribution, and reproduction in any medium, provided the original work is properly cited. 
$\left(G_{1}\right)$ There exist positive constants $\alpha_{1}$ and $\alpha_{2}$ such that

$$
\alpha_{1} \leq g, \quad\left|\nabla_{x} g\right|, g_{t}, g_{t t} \leq \alpha_{2}, \quad(x, t) \in \omega \times \mathbb{R}
$$

$\left(G_{2}\right)$ There exists a constant $k$ such that

$$
\left|g_{x_{i}}(x, t)-g_{x_{i}}(x, s)\right| \leq k|t-s|, \quad(x, t) \in \omega \times \mathbb{R}, i=1,2, \ldots, n,
$$

where $g_{t}$ denotes the partial derivative of $g$ with respect to $t, g_{x_{i}}$ denotes the partial derivative of $g$ with respect to $x_{i}, i=1,2, \ldots, n$, and $\left|\nabla_{x} g\right|$ is the Euclidian norm in $\mathbb{R}^{n}$ of the vector $\nabla_{x} g:=\left(g_{x_{1}}, \ldots, g_{x_{n}}\right)$.

In the following, $\epsilon$ denotes a positive parameter which converges to zero. Fixed $t \in \mathbb{R}$, we define the time-dependent thin domain

$$
\Omega_{t}^{\epsilon}:=\left\{(x, y) \in \mathbb{R}^{n+1}: x \in \omega, 0<y<\epsilon g(x, t)\right\} .
$$

Recalling that $\alpha_{1} \leq g(x, t) \leq \alpha_{2}$, we see that $\omega \times\left(0, \epsilon \alpha_{1}\right) \subseteq \Omega_{t}^{\epsilon} \subseteq \omega \times\left(0, \epsilon \alpha_{2}\right)$.

For each $\tau \in \mathbb{R}$ and $\epsilon \geq 0$, we set the domain

$$
Q_{\tau}^{\epsilon}:=\bigcup_{t \in(\tau, \infty)} \Omega_{t}^{\epsilon} \times\{t\}
$$

as well as the lateral boundary

$$
\Sigma_{\tau}^{\epsilon}:=\bigcup_{t \in(\tau, \infty)} \partial \Omega_{t}^{\epsilon} \times\{t\},
$$

where $\Omega_{t}^{0}:=\omega$ for all $t \in(\tau, \infty)$.

For positive values of the parameter $\epsilon$, we consider the semilinear reaction-diffusion equation

$$
\left\{\begin{array}{l}
u_{t}^{\epsilon}-\Delta u^{\epsilon}+u^{\epsilon}=f\left(u^{\epsilon}\right), \quad \text { in } Q_{\tau}^{\epsilon}, \\
\frac{\partial u^{\epsilon}}{\partial \eta_{\tau}^{\epsilon}}=0, \quad \text { on } \Sigma_{\tau}^{\epsilon}, \\
u^{\epsilon}(\cdot, \tau)=u_{\tau}^{\epsilon}, \quad \text { in } \Omega_{\tau}^{\epsilon},
\end{array}\right.
$$

where $\eta_{\tau}^{\epsilon}$ denotes the unit outward normal vector field to $\Sigma_{\tau}^{\epsilon}$, $\frac{\partial}{\partial \eta_{\tau}^{\epsilon}}$ denotes the outward normal derivative and $f: \mathbb{R} \rightarrow \mathbb{R}$ is a $C^{2}$-function with bounded derivatives up second order.

Besides, since our interest resides in the asymptotic behavior of the solutions and its dependence with respect to $\epsilon$, we will require that solutions of (1.2) are bounded for large values of the time. A natural assumption to obtain this boundedness is expressed in the following dissipative condition:

$$
\limsup _{|s| \rightarrow \infty} \frac{f(s)}{s}<0 .
$$

This implies, for any $\eta>0$, the existence of a positive constant $c_{\eta}$ such that

$$
f(s) s \leq \eta s^{2}+c_{\eta}, \quad \forall s \in \mathbb{R}
$$

We indicate to the interested reader the monograph [10, Section 4.3]. 
In the analysis of the limiting behavior of problem (1.2), it will be useful to introduce the domain $\Omega:=\omega \times(0,1)$, independent of $\epsilon$ and $t$, which is obtained from $\Omega_{t}^{\epsilon}$ by the change of coordinates

$$
\begin{aligned}
& \mathcal{T}_{t}^{\epsilon}: \Omega \rightarrow \Omega_{t}^{\epsilon}, \\
& (x, y) \mapsto(x, \epsilon g(x, t) y),
\end{aligned}
$$

which induces an isomorphism from $W^{m, p}\left(\Omega_{t}^{\epsilon}\right)$ onto $W^{m, p}(\Omega)$ defined by

$$
u \stackrel{\Phi_{t}^{\epsilon}}{\longmapsto} v:=u \circ \mathcal{T}_{t}^{\epsilon}
$$

and with partial derivatives related by

$$
\begin{aligned}
& u_{t}=v_{t}-\frac{y g_{t}}{g} v_{y}, \\
& u_{x_{i}}=v_{x_{i}}-\frac{y g_{x_{i}}}{g} v_{y}, \quad i=1, \ldots, n, \\
& u_{y}=\frac{1}{\epsilon g} v_{y} .
\end{aligned}
$$

In this new system of coordinates, equation (1.2) can be written as the following nonautonomous equation in the fixed domain $\Omega$ :

$$
\left\{\begin{array}{l}
v_{t}^{\epsilon}-\frac{1}{g} \operatorname{div} B_{\epsilon}(t) v^{\epsilon}-y \frac{g_{t}}{g} v_{y}^{\epsilon}+v^{\epsilon}=f\left(v^{\epsilon}\right), \quad \text { in } \Omega \times(\tau, \infty), \\
B_{\epsilon}(t) v^{\epsilon} \cdot \eta=0, \quad \text { on } \partial \Omega \times(\tau, \infty), \\
v^{\epsilon}(\cdot, \tau)=u_{\tau}^{\epsilon} \circ \mathcal{T}_{\tau}^{\epsilon}, \quad \text { in } \Omega,
\end{array}\right.
$$

where $\eta$ denotes the unit outward normal vector field to $\partial \Omega$, and

$$
B_{\epsilon}(t) v=\left[\begin{array}{c}
g v_{x_{1}}-y g_{x_{1}} v_{y} \\
\vdots \\
g v_{x_{n}}-y g_{x_{n}} v_{y} \\
-\sum_{i=1}^{n} y g_{x_{i}} v_{x_{i}}+\frac{1}{\epsilon^{2} g}\left(1+\sum_{i=1}^{n}\left(\epsilon y g_{x_{i}}\right)^{2}\right) v_{y}
\end{array}\right] .
$$

Next, for the reader's convenience, we argue how to derive equation (1.6) from (1.2). Consider the following variational problem: find $u^{\epsilon}(\cdot, t) \in H^{1}\left(\Omega_{t}^{\epsilon}\right)$ such that

$$
\int_{\Omega_{t}^{\epsilon}}\left[u_{t}^{\epsilon} \varphi+\nabla u^{\epsilon} \cdot \nabla \varphi+u^{\epsilon} \varphi\right] d x d y=\int_{\Omega_{t}^{\epsilon}} f\left(u^{\epsilon}\right) \varphi d x d y
$$

for all $\varphi \in C_{0}^{1}\left(\Omega_{t}^{\epsilon}\right)$. After the change of coordinates, we see by (1.5) that (1.7) is equivalent to: find $v^{\epsilon}(\cdot, t) \in H^{1}(\Omega)$ such that

$$
\begin{gathered}
\int_{\Omega}\left[\left(v_{t}^{\epsilon}-\frac{y g_{t}}{g} v_{y}^{\epsilon}\right) \tilde{\varphi}+\sum_{i=1}^{n}\left(v_{x_{i}}^{\epsilon}-\frac{y g_{x_{i}}}{g} v_{y}^{\epsilon}\right)\left(\tilde{\varphi}_{x_{i}}-\frac{y g_{x_{i}}}{g} \tilde{\varphi}_{y}\right)+\frac{1}{\epsilon^{2} g^{2}} v_{y}^{\epsilon} \tilde{\varphi}_{y}+v^{\epsilon} \tilde{\varphi}\right] g d x d y \\
\quad=\int_{\Omega}\left[g v_{t}^{\epsilon} \tilde{\varphi}+\sum_{i=1}^{n}\left(g v_{x_{i}}^{\epsilon}-y g_{x_{i}} v_{y}^{\epsilon}\right) \tilde{\varphi}_{x_{i}}+\left(-\sum_{i=1}^{n} y g_{x_{i}} v_{x_{i}}^{\epsilon}+\frac{v_{y}^{\epsilon}}{\epsilon^{2} g}\left(1+\sum_{i=1}^{n} \epsilon^{2} y^{2} g_{x_{i}}^{2}\right)\right) \tilde{\varphi}_{y}\right.
\end{gathered}
$$




$$
\begin{aligned}
& \left.+\left(-y g_{t} v_{y}^{\epsilon}+g v^{\epsilon}\right) \tilde{\varphi}\right] d x d y \\
= & \int_{\Omega}\left[g v_{t}^{\epsilon} \tilde{\varphi}+B_{\epsilon}(t) v^{\epsilon} \cdot \nabla \tilde{\varphi}+\left(-y g_{t} v_{y}^{\epsilon}+g v^{\epsilon}\right) \tilde{\varphi}\right] d x d y=\int_{\Omega} g f\left(v^{\epsilon}\right) \tilde{\varphi} d x d y
\end{aligned}
$$

for all $\tilde{\varphi} \in C_{0}^{1}(\Omega)$.

Stressing the fact that $\Omega_{t}^{\epsilon} \subset \mathbb{R}^{n+1}$ varies in accordance with the time $t$ and the small parameter $\epsilon$ collapsing itself to the lower dimensional set $\omega \subset \mathbb{R}^{n}$, one starts to suspect that $v^{\epsilon}$ tends not to depend on the variable $y$ as $\epsilon \rightarrow 0$. Therefore, we can see immediately from (1.8) that if a limiting regime for problem (1.2) exists, then it should be given by the non-autonomous problem

$$
\left\{\begin{array}{l}
v_{t}-\frac{1}{g} \sum_{i=1}^{n}\left(g v_{x_{i}}\right)_{x_{i}}+v=f(v), \quad \text { in } \omega \times(\tau, \infty), \\
\frac{\partial v}{\partial v}=0, \quad \text { on } \partial \omega \times(\tau, \infty), \\
v(\cdot, \tau)=v_{\tau}, \quad \text { in } \omega
\end{array}\right.
$$

where $v$ denotes the unit outward normal vector field to $\partial \omega$.

The paper is organized as follows. In Section 2, we introduce the abstract framework for perturbed problem (1.6) as well as for the limiting one (1.9) and we prove the existence of the associated evolution process. In Section 3 we prove the continuity of such an evolution process uniformly in compact subsets of the real line.

\section{Functional setting}

In this section we recall the definitions of suitable spaces and operators as well as some of their properties. We start recalling that $\Omega_{t}^{\epsilon}$ varies in accordance with a positive parameter $\epsilon$, collapsing itself to the lower dimensional domain $\omega$ as $\epsilon$ goes to 0 . Therefore, in order to preserve the 'relative capacity' of a mensurable subset $E \subset \Omega_{t}^{\epsilon}$, we rescale the Lebesgue measure of $E,|E|$, by a factor $1 / \epsilon$ and we are led to consider the singular measure $\rho^{\epsilon}(E):=\epsilon^{-1}|E|$. This measure has been widely considered in studies involving thin domains, e.g., $[9,11-15]$, and it allows us to introduce the Lebesgue $L^{2}\left(\Omega_{t}^{\epsilon} ; \rho^{\epsilon}\right)$ and the Sobolev $H^{1}\left(\Omega_{t}^{\epsilon} ; \rho_{\epsilon}\right)$ spaces.

It will also be convenient to consider the space $H_{\epsilon}:=H^{1}(\Omega)$ endowed with the equivalent norm

$$
\|v\|_{H_{\epsilon}}:=\left[\int_{\Omega}\left(\left|\nabla_{x} v\right|^{2}+\frac{1}{\epsilon^{2}}\left|v_{y}\right|^{2}+|v|^{2}\right) d x d y\right]^{\frac{1}{2}} .
$$

It is immediate consequence of $\left(\mathrm{G}_{1}\right)$ that the family of isomorphisms $\left\{\Phi_{t}^{\epsilon}\right\}$ satisfies

$$
\left\|\Phi_{t}^{\epsilon}\right\|_{\mathcal{L}\left(H^{1}\left(\Omega_{t}^{\epsilon} ; \rho^{\epsilon}\right), H_{\epsilon}\right)} \leq c
$$

for some positive constant $c$ independent of $\epsilon$ and $t$.

For each pair of parameters $(\epsilon, t) \in(0,1] \times \mathbb{R}$, we consider the sesquilinear form

$$
\begin{aligned}
& a_{t}^{\epsilon}: H_{\epsilon} \times H_{\epsilon} \rightarrow \mathbb{R}, \\
& a_{t}^{\epsilon}(u, v)=\int_{\Omega}\left[B_{\epsilon}(t) u \cdot \nabla v-y g_{t}(x, t) u_{y} v+g(x, t) u v\right] d x d y .
\end{aligned}
$$


Introducing the gradient operator, $\mathcal{B}_{\epsilon}(t): H^{1}(\Omega) \rightarrow\left[L^{2}(\Omega)\right]^{n+1}$,

$$
\mathcal{B}_{\epsilon}(t) v=\left(v_{x_{1}}-\frac{g_{x_{1}}(\cdot, t) y v_{y}}{g(\cdot, t)}, \ldots, v_{x_{n}}-\frac{g_{x_{n}}(\cdot, t) y v_{y}}{g(\cdot, t)}, \frac{v_{y}}{\epsilon g(\cdot, t)}\right),
$$

(2.1) can be written as

$$
a_{t}^{\epsilon}(u, v)=\int_{\Omega} g(x, t)\left(\mathcal{B}_{\epsilon}(t) u \cdot \mathcal{B}_{\epsilon}(t) v+\frac{y g_{t}(x, t) u_{y}}{g(x, t)} v+u v\right) d x d y .
$$

As the first remark, notice that under assumption $\left(\mathrm{G}_{1}\right), a_{t}^{\epsilon}$ is a continuous form and there exist positive constants $c_{1}, c_{2}$, independent of $\epsilon$ and $t$, such that

$$
c_{1}\|v\|_{H_{\epsilon}}^{2} \leq a_{t}^{\epsilon}(v, v) \leq c_{2}\|v\|_{H_{\epsilon}}^{2},
$$

for all $v \in H_{\epsilon}$ and $(\epsilon, t) \in(0, \bar{\epsilon}] \times \mathbb{R}$.

Since $H_{\epsilon}$ is densely and compactly embedded in $L^{2}(\Omega)$, the sesquilinear form $a_{t}^{\epsilon}$ yields a densely defined positive linear operator with compact resolvent, $A_{\epsilon}(t): D\left(A_{\epsilon}(t)\right) \subset$ $L^{2}(\Omega) \rightarrow L^{2}(\Omega)$, which is defined by the relation

$$
a_{t}^{\epsilon}(u, v)=\left(A_{\epsilon}(t) u, v\right)_{t}, \quad u \in D\left(A_{\epsilon}(t)\right), v \in H_{\epsilon},
$$

where $(u, v)_{t}:=\int_{\Omega} g(x, t) u v d x d y$.

By the regularity of $\partial \omega$, we have that

$$
D\left(A_{\epsilon}(t)\right)=\left\{v \in H^{2}(\Omega): B_{\epsilon}(t) v \cdot \eta=0\right\},
$$

is independent of $\epsilon$. Moreover,

$$
A_{\epsilon}(t) v=-\frac{1}{g(\cdot, t)} \operatorname{div} B_{\epsilon}(t) v-y \frac{g_{t}(\cdot, t)}{g(\cdot, t)} v_{y}+v, \quad v \in D\left(A_{\epsilon}(t)\right) .
$$

Multiplying (1.6) by $\varphi \in H^{1}(\Omega)$ and integrating by parts, we get

$$
\left(v_{t}^{\epsilon}, \varphi\right)_{t}+a_{t}^{\epsilon}\left(v^{\epsilon}, \varphi\right)=\left(f\left(v^{\epsilon}\right), \varphi\right)_{t} .
$$

Therefore we can write equation (1.6) as an abstract evolution equation

$$
\frac{d v^{\epsilon}}{d t}(t)+A_{\epsilon}(t) v^{\epsilon}(t)=f^{e}\left(v^{\epsilon}(t)\right)
$$

where $f^{e}$ is the Nemitskii operator (composition operator) associated to $f$.

Combining assumptions $\left(\mathrm{G}_{1}\right)$ and $\left(\mathrm{G}_{2}\right)$, we also obtain that

$$
\left|a_{t}^{\epsilon}(u, v)-a_{s}^{\epsilon}(u, v)\right| \leq k_{1}|t-s|\|u\|_{H_{\epsilon}}\|v\|_{H_{\epsilon}}
$$

for some constant $k_{1}$ independent of $\epsilon, t, s \in \mathbb{R}$, and $u, v \in H_{\epsilon}$. Therefore, thanks to [16, Theorem 5.4.2], there exists a unique solution of the linear homogeneous problem

$$
\left\{\begin{array}{l}
\frac{d v^{\epsilon}}{d t}(t)+A_{\epsilon}(t) v^{\epsilon}(t)=0, \quad t>\tau \in \mathbb{R} \\
v^{\epsilon}(\tau)=v_{\tau}^{\epsilon} \in H_{\epsilon} .
\end{array}\right.
$$


This allows us to consider, for each value of the parameter $\epsilon$, each initial time $\tau \in \mathbb{R}$ and each initial data $v_{\tau}^{\epsilon} \in H_{\epsilon}$, the solution $v^{\epsilon}\left(\cdot, \tau, v_{\tau}^{\epsilon}\right) \in C^{1}\left([\tau, \infty) ; H_{\epsilon}\right)$ of (2.5). This gives rise to a linear process $\left\{L_{\epsilon}(t, \tau), t \geq \tau\right\} \subset \mathcal{L}\left(H_{\epsilon}\right)$ defined by $L_{\epsilon}(t, \tau) v_{\tau}^{\epsilon}:=v^{\epsilon}\left(t, \tau, v_{\tau}^{\epsilon}\right)$. We notice that (2.5) is the abstract Cauchy problem associated to equation (1.6) in the case $f \equiv 0$.

Since we are assuming the nonlinearity $f \in C^{2}(\mathbb{R} ; \mathbb{R})$ bounded as well as its derivatives up second order, local existence of the nonlinear counterpart is guaranteed by [16, Theorem 6.6.1], i.e., writing problem (1.6) as

$$
\left\{\begin{array}{l}
\frac{d v^{\epsilon}}{d t}(t)+A_{\epsilon}(t) v^{\epsilon}(t)=f^{e}\left(v^{\epsilon}(t)\right), \\
v^{\epsilon}(\tau)=v_{\tau}^{\epsilon} \in H_{\epsilon},
\end{array}\right.
$$

there exist time $T_{\tau}>0$ and a unique solution $v^{\epsilon}\left(\cdot, \tau, v_{\tau}^{\epsilon}\right) \in C^{1}\left(\left[\tau, \tau+T_{\tau}\right] ; H_{\epsilon}\right)$ of (2.6). Under assumption (1.3) on the nonlinearity $f$, one can show that actually $v^{\epsilon}\left(\cdot, \tau, v_{\tau}^{\epsilon}\right) \in$ $C^{1}\left([\tau, \infty) ; H_{\epsilon}\right)$. Further details can be found in [10, Section 4.3] and [17, Theorem 3.2].

Similarly to the linear case, this allows us to consider, for each value of the parameter $\epsilon$, each initial time $\tau \in \mathbb{R}$, and each initial data $v^{\epsilon} \in H_{\epsilon}$, the (nonlinear) evolution process $\left\{S_{\epsilon}(t, \tau): t \geq \tau\right\}$ in the state space $H_{\epsilon}$ defined by $S_{\epsilon}(t, \tau) v^{\epsilon}:=v^{\epsilon}\left(t, \tau, v^{\epsilon}\right)$. According to [16],

$$
S_{\epsilon}(t, \tau) v^{\epsilon}=L_{\epsilon}(t, \tau) v^{\epsilon}+\int_{\tau}^{t} L_{\epsilon}(t, s) f^{e}\left(S_{\epsilon}(s, \tau) v^{\epsilon}\right) d s, \quad \forall t \geq \tau \in \mathbb{R}
$$

where $\left\{L_{\epsilon}(t, \tau): t \geq \tau \in \mathbb{R}\right\}$ is the linear evolution process associated to homogeneous problem (2.5).

For the reader's convenience, we recall the definition of an evolution process in a Banach space.

Definition 2.1 We say that a family of maps $\{S(t, \tau): t \geq \tau \in \mathbb{R}\}$ from a Banach space $\mathcal{X}$ into itself is an evolution process if

(i) $S(\tau, \tau)=I$ (identity operator in $\mathcal{X}$ ) for any $\tau \in \mathbb{R}$,

(ii) $S(t, \sigma) S(\sigma, \tau)=S(t, \tau)$ for any $t \geq \sigma \geq \tau$,

(iii) $(t, \tau) \mapsto S(t, \tau) v$ is continuous for all $t \geq \tau$ and $v \in \mathcal{X}$.

\subsection{Limiting consideration}

For each $t \in \mathbb{R}$, we consider the sesquilinear form

$$
a_{t}^{0}: H^{1}(\omega) \times H^{1}(\omega) \rightarrow \mathbb{R}
$$

defined by

$$
a_{t}^{0}(u, v)=\int_{\omega} g(x, t)(\nabla u \cdot \nabla v+u v) d x
$$

With this definition, we immediately see that

$$
\alpha_{1}\|v\|_{H^{1}(\omega)}^{2} \leq a_{t}^{0}(v, v) \leq \alpha_{2}\|v\|_{H^{1}(\omega)}^{2}
$$

for all $v \in H^{1}(\omega)$. 
Similarly to the previous section, since that $H^{1}(\omega)$ is densely and compactly embedded in $L^{2}(\omega), a_{t}^{0}$ gives rise to a densely defined positive linear operator with compact resolvent, $A_{0}(t): D\left(A_{0}(t)\right) \subset L^{2}(\omega) \rightarrow L^{2}(\omega)$, defined by the relation

$$
a_{t}^{0}(u, v)=\left(\left(A_{0}(t) u, v\right)\right)_{t}, \quad u \in D\left(A_{0}(t)\right), v \in H^{1}(\omega)
$$

where $((u, v))_{t}:=\int_{\omega} g(x, t) u v d x, u, v \in L^{2}(\omega)$.

By the regularity of $\partial \omega$,

$$
D\left(A_{0}(t)\right)=\left\{v \in H^{2}(\omega): \nabla v \cdot v=0\right\},
$$

and is independent of $t$. Moreover,

$$
A_{0}(t) v=-\frac{1}{g(\cdot, t)} \sum_{i=1}^{n}\left(g(\cdot, t) v_{x_{i}}\right)_{x_{i}}+v, \quad v \in D\left(A_{0}(t)\right)
$$

By $\left(\mathrm{G}_{1}\right)-\left(\mathrm{G}_{2}\right)$ there exists a constant $k_{2}$ (independent of $t$ ) such that

$$
\left|a_{t}^{0}(u, v)-a_{t}^{0}(u, v)\right| \leq k_{2}|t-s|\|u\|_{H^{1}(\omega)}\|v\|_{H^{1}(\omega)}
$$

for all $t, s \in \mathbb{R}$ and $u, v \in H^{1}(\omega)$.

Therefore, writing equation (1.9) as an abstract evolution equation

$$
\left\{\begin{array}{l}
\frac{d v^{0}}{d t}(t)+A_{0}(t) v^{0}(t)=f^{e}\left(v^{0}(t)\right), \quad t>\tau \in \mathbb{R}, \\
v^{0}(\tau)=v_{\tau}^{0} \in H^{1}(\omega),
\end{array}\right.
$$

we can define an evolution process, $\left\{S_{0}(t, \tau): t \geq \tau\right\}$, in the state space $H^{1}(\omega)$ setting $S_{0}(t, \tau) v^{0}:=v^{0}\left(t, \tau, v^{0}\right)$. We notice that

$$
S_{0}(t, \tau) v^{0}=L_{0}(t, \tau) v^{0}+\int_{\tau}^{t} L_{0}(t, s) f^{e}\left(S_{0}(s, \tau) v^{0}\right) d s, \quad \forall t \geq \tau \in \mathbb{R},
$$

where $\left\{L_{0}(t, \tau): t \geq \tau \in \mathbb{R}\right\}$ is the evolution process associated to the linear homogeneous counterpart.

Now we have the elements to state our main result.

Theorem 2.2 Under assumptions $\left(\mathrm{G}_{1}\right),\left(\mathrm{G}_{2}\right)$ on the profile $g \in C^{2}(\bar{\omega} \times \mathbb{R} ; \mathbb{R})$, and assuming that the nonlinearity $f \in C^{2}(\mathbb{R} ; \mathbb{R})$ has bounded derivatives up second order and satisfies (1.3), equations (2.6) and (2.10) generate evolution processes $\left\{S_{\epsilon}(t, \tau): t \geq \tau\right\}$ and $\left\{S_{0}(t, \tau)\right.$ : $t \geq \tau\}$ in $H_{\epsilon}$ and $H^{1}(\omega)$, respectively. Moreover, given $v^{\epsilon} \in H_{\epsilon}$ and $v^{0} \in H^{1}(\omega)$ such that $v^{\epsilon} \stackrel{\epsilon \rightarrow 0}{\longrightarrow} E v^{0}$ in $L^{2}(\Omega)$, then

$$
\left\|S_{\epsilon}(t, \tau) v^{\epsilon}-E S_{0}(t, \tau) v^{0}\right\|_{H_{\epsilon}} \stackrel{\epsilon \rightarrow 0}{\longrightarrow} 0,
$$

uniformly for $(t, \tau), t \geq \tau$, in bounded subsets of $\mathbb{R}^{2}$, where the extension operator $E$ is defined in (3.2). 


\section{Convergence results}

Due to the nature of this specific kind of singular perturbations, it is natural to introduce the following operators in order to compare functions defined in the different domains $\Omega$ and $\omega$.

(Average projector)

$$
\begin{aligned}
& M: L^{p}(\Omega) \rightarrow L^{p}(\omega), \\
& (M u)(x)=\int_{0}^{1} u(x, y) d y .
\end{aligned}
$$

(Extension operator)

$$
\begin{aligned}
& E: L^{p}(\omega) \rightarrow L^{p}(\Omega), \\
& (E u)(x, y)=u(x) .
\end{aligned}
$$

Notice that the extension operator $E$ maps the family of spaces $W^{1, p}(\omega)$ into $W^{1, p}(\Omega)$.

Lemma 3.1 If $\left\{f^{\epsilon}\right\}$ is a bounded family in $L^{2}(\Omega)$, then $\left\{A_{\epsilon}(t)^{-1} f^{\epsilon}\right\}$ is a bounded family in $H_{\epsilon}$.

Proof For each $t$ fixed, we set $u^{\epsilon}:=A_{\epsilon}(t)^{-1} f^{\epsilon} \in H_{\epsilon}$. Therefore

$$
a_{t}^{\epsilon}\left(u^{\epsilon}, v\right)=\left(f^{\epsilon}, v\right)_{t}, \quad \forall v \in H_{\epsilon} .
$$

In particular, taking $v=u^{\epsilon}$ as a test function, it follows from (2.2) and Hölder's inequality that

$$
c_{1}\left\|u^{\epsilon}\right\|_{H_{\epsilon}}^{2} \leq a_{t}^{\epsilon}\left(u^{\epsilon}, u^{\epsilon}\right) \leq \alpha_{2}\left\|f^{\epsilon}\right\|_{L^{2}(\Omega)}\left\|u^{\epsilon}\right\|_{H_{\epsilon}},
$$

which proves the result.

The following lemma describes the behavior of the operators $A_{\epsilon}(t)^{-1}$ as $\epsilon \rightarrow 0$.

Lemma 3.2 Let $\left\{f^{\epsilon}\right\}$ be a bounded family in $L^{2}(\Omega)$. If $M f^{\epsilon} \stackrel{\epsilon \rightarrow 0}{\rightarrow} \hat{f}$ weakly in $L^{2}(\omega)$, then

$$
\left\|A_{\epsilon}(t)^{-1} f^{\epsilon}-E A_{0}(t)^{-1} \hat{f}\right\|_{H_{\epsilon}} \stackrel{\epsilon \rightarrow 0}{\longrightarrow} 0
$$

uniformly in $t$ in bounded subsets of $\mathbb{R}$.

Proof If $u \in H_{\epsilon}$ and $\hat{v} \in H^{1}(\omega)$, then

$$
a_{t}^{\epsilon}(u, E \hat{v})=a_{t}^{0}(M u, \hat{v})-\int_{\Omega} y u_{y}\left(\nabla_{x} g \cdot \nabla_{x} \hat{v}+g_{t} \hat{v}\right) d x d y .
$$

Besides, for $u \in H_{\epsilon}$ and $\hat{v} \in L^{2}(\omega)$, we have that

$$
a_{t}^{\epsilon}\left(u, A_{\epsilon}(t)^{-1} E \hat{v}\right)=(u, E \hat{v})_{t}=\int_{\omega} g(t, x) \hat{v} M u d x=((M u, \hat{v}))_{t}=a_{t}^{0}\left(M u, A_{0}(t)^{-1} \hat{v}\right) .
$$


Therefore,

$$
\begin{aligned}
& \left|a_{t}^{\epsilon}\left(u, A_{\epsilon}(t)^{-1} E \hat{v}-E A_{0}(t)^{-1} \hat{v}\right)\right| \\
& =\mid a_{t}^{0}\left(M u, A_{0}(t)^{-1} \hat{v}\right)-a_{t}^{0}\left(M u, A_{0}(t)^{-1} \hat{v}\right) \\
& \quad-\int_{\Omega} y u_{y}\left(\nabla_{x} g \cdot \nabla_{x} A_{0}(t)^{-1} \hat{v}+g_{t} A_{0}(t)^{-1} \hat{v}\right) d x d y \mid \\
& \leq c\left\|u_{y}\right\|_{L^{2}(\Omega)}\left\|A_{0}(t)^{-1} \hat{v}\right\|_{H^{1}(\omega)} \\
& \leq c \epsilon\|u\|_{H_{\epsilon}}\left\|A_{0}(t)^{-1} \hat{v}\right\|_{H^{1}(\omega)} .
\end{aligned}
$$

Taking $u=A_{\epsilon}(t)^{-1} E \hat{v}-E A_{0}(t)^{-1} \hat{v}$ in the last inequality, we derive the result from (2.2) recalling Lemma 3.1.

Proof of Theorem 2.2 Recalling that the nonlinear term $f$ has bounded derivatives up second order, $M \varphi \in H^{1}(\omega)$ whenever $\varphi \in H^{1}(\Omega)$ and $E S_{0}(t, \tau) v^{0}$ does not depend on the variable $y$, we have that $\omega_{\epsilon}(t):=S_{\epsilon}(t, \tau) v^{\epsilon}-E S_{0}(t, \tau) v^{0}$ satisfies the following energy estimate:

$$
\begin{aligned}
\left(\frac{d}{d t} \omega_{\epsilon}(t), \varphi\right)_{t}+a_{t}^{\epsilon}\left(\omega_{\epsilon}(t), \varphi\right) & =\left(f^{e}\left(S_{\epsilon}(t, \tau) v^{\epsilon}\right)-E f^{e}\left(S_{0}(t, \tau) v^{0}\right), \varphi\right)_{t} \\
& \leq L\left\|\omega_{\epsilon}(t)\right\|_{L^{2}(\Omega)}\|\varphi\|_{L^{2}(\Omega)}
\end{aligned}
$$

for some positive constant $L$ (not dependent on $\epsilon$ and $t$ ) and for all $\varphi \in H^{1}(\Omega)$. In particular, taking $\varphi=\omega_{\epsilon}(t)$, we obtain that

$$
\frac{1}{2} \frac{d}{d t}\left\|\omega_{\epsilon}(t)\right\|_{L^{2}(\Omega)}^{2}+\left\|\omega_{\epsilon}(t)\right\|_{H_{\epsilon}}^{2} \leq L\left\|\omega_{\epsilon}(t)\right\|_{L^{2}(\Omega)}^{2} .
$$

It follows from Gronwall's inequality that

$$
\left\|\omega_{\epsilon}(t)\right\|_{L^{2}(\Omega)}^{2} \leq \tilde{c}\left\|v^{\epsilon}-E v^{0}\right\|_{L^{2}(\Omega)}^{2}
$$

in compact subsets of $\mathbb{R}$.

To conclude, we need to ensure that $\left\{\left\|A_{\epsilon}(t) S_{\epsilon}(t, \tau) v^{\epsilon}\right\|_{L^{2}(\Omega)}\right\}$ is uniformly bounded with respect to the parameter $\epsilon$, which is equivalent to ensuring that $\left\{\left\|S_{\epsilon}(t, \tau) v^{\epsilon}\right\|_{H_{\epsilon}}\right\}$ is uniformly bounded with respect to $\epsilon$. In doing this, we are inspired by the previous argument. In fact, since

$$
\left(\frac{d}{d t} S_{\epsilon}(t, \tau) v^{\epsilon}, S_{\epsilon}(t, \tau) v^{\epsilon}\right)_{t}+a_{t}^{\epsilon}\left(S_{\epsilon}(t, \tau) v^{\epsilon}, S_{\epsilon}(t, \tau) v^{\epsilon}\right)=\left(f^{e}\left(S_{\epsilon}(t, \tau) v^{\epsilon}\right), S_{\epsilon}(t, \tau) v^{\epsilon}\right){ }_{t}
$$

we obtain that

$$
\alpha_{1} \frac{d}{d t}\left\|S_{\epsilon}(t, \tau) v^{\epsilon}\right\|_{L^{2}(\Omega)}^{2}+c_{1}\left\|S_{\epsilon}(t, \tau) v^{\epsilon}\right\|_{H_{\epsilon}}^{2} \leq \alpha_{2} L\left\|S_{\epsilon}(t, \tau) v^{\epsilon}\right\|_{L^{2}(\Omega)}^{2}
$$

which shows that $\left\{\left\|S_{\epsilon}(t, \tau) v^{\epsilon}\right\|_{L^{2}(\Omega)}^{2}\right\}$ is uniformly bounded. Therefore

$$
\alpha_{1} \frac{d}{d t}\left\|S_{\epsilon}(t, \tau) v^{\epsilon}\right\|_{L^{2}(\Omega)}^{2}+c_{1}\left\|S_{\epsilon}(t, \tau) v^{\epsilon}\right\|_{H_{\epsilon}}^{2} \leq M_{1}
$$

for some constant $M_{1}$ independent of $\epsilon$. 
Finally,

$$
\begin{aligned}
\left\|\omega_{\epsilon}(t)\right\|_{H_{\epsilon}}^{2} & \leq c_{2} a_{t}^{\epsilon}\left(\omega_{\epsilon}(t), \omega_{\epsilon}(t)\right) \\
& =c_{2}\left(A_{\epsilon}(t) \omega_{\epsilon}(t), \omega_{\epsilon}(t)\right)_{t} \\
& \leq c_{2}\left\|\omega_{\epsilon}(t)\right\|_{L^{2}(\Omega)}\left[\left\|A_{\epsilon}(t) S_{\epsilon}(t, \tau) v^{\epsilon}\right\|_{L^{2}(\Omega)}+\left\|A_{0}(t) S_{0}(t, \tau) v^{0}\right\|_{L^{2}(\omega)}\right] \\
& \leq \tilde{c}_{2}\left\|v^{\epsilon}-E v^{0}\right\|_{L^{2}(\Omega)}^{2}
\end{aligned}
$$

for $t$ in compact subsets of $\mathbb{R}$.

\section{Competing interests}

The authors declare that they have no competing interests.

\section{Authors' contributions}

We declare that all authors collaborated and dedicated the same amount of time in order to perform this article.

\section{Acknowledgements}

This paper is dedicated in memoriam to José Carlos Arcuri da Silva. RPS is partially supported by FAPESP \#2012/06753-8, FUNDUNESP \# 0135812 and FUNDUNESP_PROPe \#0019/008/13, Brazil. The authors thank the referees for their careful reading and suggestions which led to an improvement of the work.

\section{Received: 1 July 2013 Accepted: 25 October 2013 Published: 20 Nov 2013}

\section{References}

1. Anh, CT, Toan, ND: Pullback attractors for nonclassical diffusion equations in noncylindrical domains. Int. J. Math. Math. Sci. 2012, Article ID 875913 (2012)

2. Bayada, G, Chambat, M, Ciuperca, I: Comportement asymptotique d'un fluide dans un domaine mince variable en temps. C. R. Math. Acad. Sci. Paris 326, 265-268 (1998)

3. Clark, HR, Rincon, MA, Rodrigues, RD: Beam equation with weak-internal damping in domain with moving boundary, Appl. Numer. Math. 47, 139-157 (2003)

4. Kloeden, PE, Marín-Rubio, P, Real, J: Pullback attractors for a semilinear heat equation in a non-cylindrical domain. J. Differ. Equ. 244, 2062-2091 (2008)

5. Kloeden, PE, Real, J, Sun, C: Pullback attractors for a semilinear heat equation on time-varying domains. J. Differ. Equ. $246,4702-4730$ (2009)

6. Menezes, SB, Limaco, J, Medeiros, LA: Remarks on null controllability for semilinear heat equation in moving domains. Electron. J. Qual. Theory Differ. Equ. 16, 1-32 (2003)

7. Carvalho, AN, Langa, JA, Robinson, JC: Attractors for Infinite-Dimensional Non-Autonomous Dynamical Systems. Applied Mathematical Sciences, vol. 182. Springer, Berlin (2012)

8. Chepyzhov, W, Vishik, Ml: Attractors for Equations of Mathematical Physics. Colloquium Publications, vol. 49. Am. Math. Soc., Providence (2002)

9. Hale, JK, Raugel, G: Reaction-diffusion equation on thin domains. J. Math. Pures Appl. 71, 33-95 (1992)

10. Hale, JK: Asymptotic Behavior of Dissipative Systems. Mathematical Surveys and Monographs, vol. 25. Am. Math. Soc., Providence (1988)

11. Arrieta, JM, Carvalho, AN, Pereira, MC, Silva, RP: Semilinear parabolic problems in thin domains with a highly oscillatory boundary. Nonlinear Anal. 74, 5111-5132 (2011)

12. Pereira, MC, Silva, RP: Error estimates for a Neumann problem in highly oscillating thin domains. Discrete Contin. Dyn. Syst., Ser. A 33(2), 803-817 (2013)

13. Prizzi, M, Rybakowski, KP: The effect of domain squeezing upon the dynamics of reaction-diffusion equations. J. Differ. Equ. 173, 271-320 (2001)

14. Raugel, G: Dynamics of Partial Differential Equations on Thin Domains. Lecture Notes in Mathematics, vol. 1609. Springer, Berlin (1995)

15. Silva, RP: A note on resolvent convergence on a thin domain. Bull. Aust. Math. Soc. (2013). doi:10.1017/S0004972713000555

16. Tanabe, H: Equations of Evolution. Pitman, London (1979)

17. Carbone, VL, Nascimento, MJD, Schiabel-Silva, K, Silva, RP: Pullback attractors for a singularly nonautonomous plate equation. Electron. J. Differ. Equ. 77, 1-13 (2011)

10.1186/1687-2770-2013-248

Cite this article as: Pereira and Silva: Reaction-diffusion equations in a noncylindrical thin domain. Boundary Value Problems 2013, 2013:248 